\title{
Speckle Reduction in Carotid Artery Images using Dual Tree Complex Wavelet Transform
}

\author{
V. N. Prudhvi Raj \\ Associate Professor \\ Department of EIE \\ VR Siddhartha Engineering College, Vijayawada
}

\author{
G. Geetha \\ Assistant Professor \\ Department of IT \\ VR Siddhartha Engineering College, Vijayawada
}

\begin{abstract}
Carotid artery disease is a condition in which the carotid arteries become narrowed or blocked. When the arteries become narrowed, the condition is called carotid stenosis. The carotid arteries provide the main blood supply to the brain. Carotid artery disease occurs when sticky, fatty substances called plaque build-up in the inner lining of the arteries. The plaque may slowly block or narrow the carotid artery or cause a clot (thrombus) to form more suddenly. Clots can lead to stroke. Imaging techniques have long been used for assessing and treating cardiac and carotid disease. B-mode ultrasound imaging or intravascular ultrasound (IVUS) has emerged, and it is widely used for visualizing carotid plaques and assessing plaque characteristics that are related to the onset of neurological symptoms. In Medical diagnosis operations such as feature extraction and object recognition will play the key role. These tasks will become difficult if the images are corrupted with noises. So the development of effective algorithms for noise removal became an important research area in present days. Developing Image denoising algorithms is a difficult task since fine details in a medical image embedding diagnostic information should not be destroyed during noise removal. The ultrasound imaging suffers from speckle noise. Many of the wavelet based denoising algorithms use DWT (Discrete Wavelet Transform) in the decomposition stage which is suffering from shift variance and lack of directionality. To overcome this in this paper we are using the denoising method which uses dual tree complex wavelet transform to decompose the image and we performed the shrinkage operation to eliminate the noise from the noisy image. In the shrinkage step we used semi-soft thresholding operator along with traditional hard and soft thresholding operators and verified the suitability of dual tree complex wavelet transform for the denoising of ultrasound images. The results proved that the denoised image using DTCWT (Dual Tree Complex Wavelet Transform) have a better balance between smoothness and accuracy than the DWT and less redundant than UDWT (Undecimated Wavelet Transform). We used the SSIM (Structural similarity index measure) along with PSNR to assess the quality of denoised images . [1],[2],[3]
\end{abstract}

\section{Keywords}

Carotid artery, Discrete Wavelet Transform, Undecimated Wavelet Transform, Dual Tree Complex Wavelet Transform

\section{INTRODUCTION}

Medical information, composed of clinical data, images and other physiological signals, has become an essential part of a patient's care, whether during screening, the diagnostic stage or the treatment phase. Over the past three decades, rapid developments in information technology (IT) and Medical Instrumentation has facilitated the development of digital medical imaging. This development has mainly concerned Computed Tomography (CT), Magnetic Resonance Imaging (MRI), the different digital radiological processes for vascular, cardiovascular and contrast imaging, mammography, diagnostic ultrasound imaging, nuclear medical imaging with Single Photon Emission Computed Tomography (SPECT) and Positron Emission Tomography (PET). All these processes are producing ever-increasing quantities of images. These images are different from typical photographic images primarily because they reveal internal anatomy as opposed to an image of surfaces [3].

Spatial filters are traditional means of removing noise from images and signals. Spatial filters usually smooth the data to reduce the noise, and also blur the data. Several new techniques have been developed in the last few years that improve on spatial filters by removing the noise more effectively while preserving the edges in the data. Some of these techniques used the ideas from partial differential equations and computational fluid dynamics such as level set methods, total variation methods, non-linear isotropic and anisotropic diffusion, other techniques combine impulse removal filters with local adaptive filtering in the transform domain to remove not only white and mixed noise, but also their mixtures. In order to reduce the noise present in medical images many techniques are available like digital filters (FIR or IIR), adaptive filtering methods etc. However, digital filters and adaptive methods can be applied to signal whose statistical characteristics are stationary in many cases. Recently the wavelet transform has been proven to be useful tool for non-stationary signal analysis [4],[5],[6],[7],[8],[9].

\subsection{Carotid artery stenosis}

Carotid artery disease is a condition in which the carotid arteries become narrowed or blocked. When the arteries become narrowed, the condition is called carotid stenosis. The carotid arteries provide the main blood supply to the brain. They are located on each side 
of neck. Carotid artery disease occurs when sticky, fatty substances called plaque build-up in the inner lining of the arteries. The plaque may slowly block or narrow the carotid artery or cause a clot (thrombus) to form more suddenly. Clots can lead to stroke [10].

Ultrasound imaging is used to detect the formation of plaque in carotid artery. The early detection of plaque will help to prevent the stroke. But the ultrasound image is suffering from speckle noise. Speckle is a multiplicative noise that degrades image quality and the visual evaluation in ultrasound and SAR imaging. This necessitates the need for robust despeckling techniques in a wide spectrum of the aforementioned imaging applications. Despeckle filtering applications has been a rapidly emerging research area in recent years. The objective of our work is develop new denoising algorithms to detect the presence of plaque which is dominated by the speckle noise in ultrasound images to diagnose the risk of stroke and compare the efficiency of the same with the existing algorithms.

\section{DISCRETE WAVELET TRANSFORM}

The DWT of a signal $x(n)$ is calculated by passing it through a series of filters. First the samples are passed through a low pass filter with impulse response $g(n)$ resulting in a convolution of the two:

$$
y[n]=(x * g)[n]=\sum_{k=-\infty}^{\infty} x(k) g(n-k)
$$

The signal is also decomposed simultaneously using a high-pass filter with impulse respone $h(n)$. The outputs of the highpass filter are detail coefficients and the outputs of the lowpass filter are approximation coefficients. It is important that the two filters are related to each other and they are known as a quadrature mirror filter. Since half the frequencies of the signal have now been removed, half the samples can be discarded according to Nyquist's rule. The filter outputs are then subsampled by 2

$$
\begin{gathered}
y_{\text {low }}[n]=\sum_{k=-\infty}^{\infty} x(k) g(2 n-k) \\
y_{\text {high }}[n]=\sum_{k=-\infty}^{\infty} x(k) h(2 n+1-k)
\end{gathered}
$$

This decomposition has halved the time resolution since only half of each filter output characterises the signal. However, each output has half the frequency band of the input so the frequency resolution has been doubled. This decomposition is repeated to further increase the frequency resolution and the approximation coefficients decomposed with high and low pass filters and then down-sampled [11.

\section{$2.12 D$ DWT}

2D DWT can be implemented by applying the 1D DWT along the rows of an image first and applying then on the columns of an image. When a wavelet transform is applied to an image which is a $2 \mathrm{D}$ signal it decomposes the image into four subbands. The LL band contains the approximation coefficients, LH band contains horizontal details, HL band contains vertical details and $\mathrm{HH}$ band will contain the diagonal details [11].

\section{UNDECIMATED WAVELET TRANSFORM}

Decimation of the wavelet coefficients is an intrinsic property of the discrete wavelet transform (DWT). The decimation step removes every other of the coefficients of the current level. Thus the computation of the wavelet transform is faster and more compact in terms of storage space. More importantly, the transformed signal can be perfectly reconstructed from the remaining coefficients. Unfortunately, the decimation is causing shift variance of the wavelet transform [12].

In order to achieve shift- invariance, researches from different fields and having various goals have invented several wavelet transform algorithms. This type of transforms is known under the common name undecimated wavelet transform (UWT).

Unlike the discrete wavelet transform (DWT), which downsamples the approximation coefficients and detail coefficients at each decomposition level, the undecimated wavelet transform (UWT) does not incorporate the downsampling operations. Thus, the approximation coefficients and detail coefficients at each level are the same length as the original signal. The UWT upsamples the coefficients of the lowpass and highpass filters at each level. The upsampling operation is equivalent to dilating wavelets. The resolution of the UWT coefficients decreases with increasing levels of decomposition. By comparing the UWT with the DWT, the UWT has some unique features, Translation invariance, better denoising capability, better peak detection capability [12].

\section{DUAL TREE COMPLEX WAVELET TRANSFORM}

The dual tree complex wavelet transform is directionally selective and shift invariant in two and higher dimensions. The dual tree complex wavelet transform introduces the redundancy by a factor of $2^{d}$ for $d$ dimensions which is lower than the redundancy introduced by UDWT (Undecimated Wavelet Transform)Since last 20 years DWT (Discrete Wavelet Transform) has proven excellent tool for analysis of one dimensional signal's by replacing the Fourier Transform's infinitely oscillating sinusoidal basis functions with a set of locally oscillating functions called wavelets. But Its performance is poor in the analysis of complex and modulated signals such as radar, speech, music, higher dimensional medical and geophysics data. In these areas the complex wavelet transform will give a better performance than critically sampled DWT [13],[14].

The Discrete wavelet transform is suffering from four shortcomings

Oscillations: The wavelets are bandpass functions, so the coefficients of wavelet transform will oscillate positive and negative around singularities. This will complicate signal modelling and singularity extraction.

Shift Variance: A Small shift in the signal of interest will perturb the wavelet coefficient oscillation pattern around singularities. The wavelet transform coefficients of signal $x(t)$ and its shifted version $x(t-d)$ are not same.

Aliasing: The wavelet coefficient processing methods like thresholding, filtering and quantization etc. will upset the balance between the Analysis and synthesis filter banks so the artifacts will present in the reconstructed signal. 
Lack of Directionality: DWT will process the point singularities effectively but it can be difficult to model and process the geometric features like lines, edges and ridges.

These four short comings can be minimised by using dual tree wavelet transform in processing the medical images than the traditional DWT and UDWT. UDWT is shift invariant but the redundancy introduced is more than the dual tree wavelet transforms. There are three wavelets associated with the 2D wavelet transform. The following figure illustrates three wavelets as gray scale images.

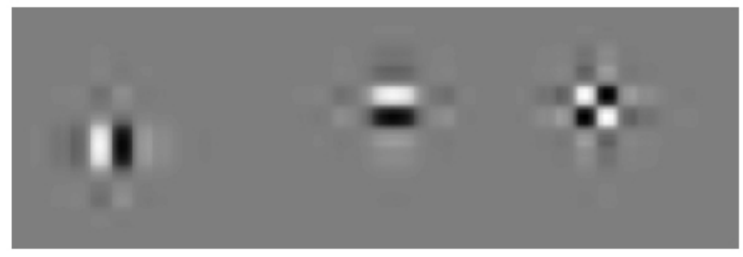

Fig. 1: 2D Wavelets

The first two wavelets are oriented in the vertical and horizontal directions and the third wavelet does not have a dominant orientation. The third wavelet mixes two diagonal orientations, which gives rise to the checkerboard artifact. The 2D DWT is poor at isolating the two diagonal orientations i.e it can't distinguish $+45^{\circ}$ line and $-45^{\circ}$. The complex 2-D dual-tree DWT have wavelets in six distinct directions as shown in the following figure. There are two wavelets in each direction. In each direction, one of the two wavelets can be interpreted as the real part of a complex-valued 2D wavelet, and the other wavelet as the imaginary part of a complexvalued $2 \mathrm{D}$ wavelet.

2D Dual-Tree Complex Wavelets

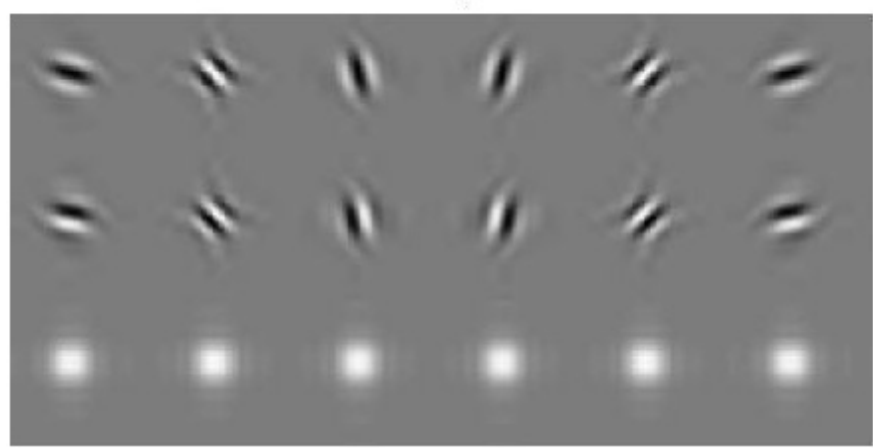

Fig. 2: 2D Dual Tree Complex Wavelets

The dual-tree complex DWT of a signal $\mathrm{x}$ is computed using two critically-sampled DWTs in parallel on the same data as shown in the following figure. If the same filters used in the upper tree and lower tree nothing is gained. So the filters in this structure will designed in a specific way that the subbands of upper DWT is interpreted as real part of complex wavelet transform and the lower tree as imaginary part. The transform is expansive by a factor 2 and shift invariant. The complex 2D wavelet is shown in the following figure.

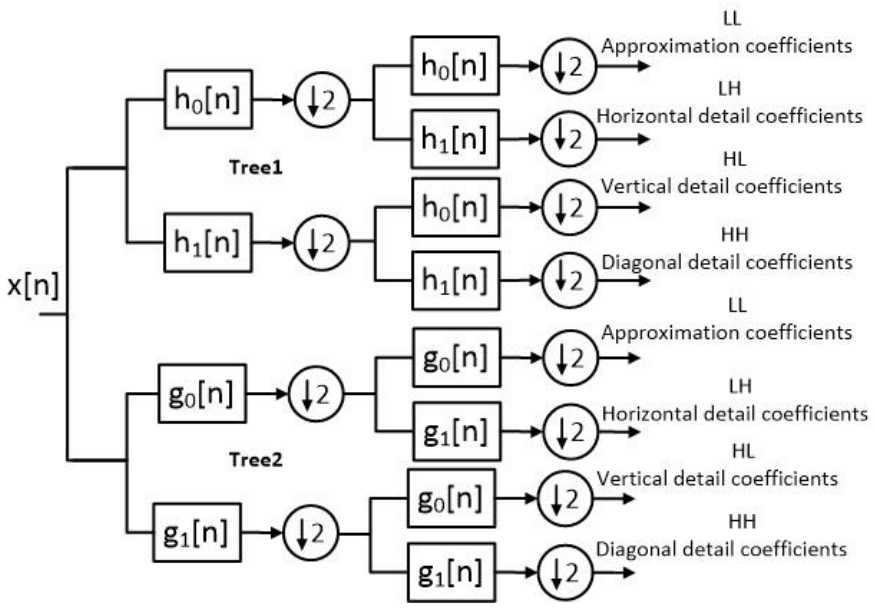

Fig. 3: Dual Tree Wavelet Implementation

There are various methods to design the filters for dual tree complex wavelet transform. The detailed study of filter design is found in the article "The Dual-Tree Complex Wavelet Transform" by Nick G. Kingsbury [9]. The filters must satisfy the desired properties such as approximate half sample property, Perfect Reconstruction (Orthogonal or Biorthogonal), Finite support (FIR filters), and Vanishing moments/good stop band, Linear phase.

\section{DENOISING ALGORITHM}

The wavelet shrinkage is a signal denoising technique based on the idea of thresholding the wavelet coefficients. Wavelet coefficients having small absolute value are considered to encode mostly noise and very fine details of the signal. In contrast, the important information is encoded by the coefficients having large absolute value. Removing the small absolute value coefficients and then reconstructing the signal should produce signal with lesser amount of noise. The wavelet shrinkage approach can be summarized as follows.

1. Apply the forward transform on the noisy image. There are various wavelet families such as Haar, Daubechies, Coieflets, Symlets, biorthogonal etc. developed in the literature. In this paper we used dubechies, symlets for the 2D DWT and UDWT. We used the dual tree complex wavelet transform developed by Ivan W Selesnick and Nick G Kingsbury. The image can be divided into number of multiresolution levels. The maximum number of possible decomposition levels is $n=\log _{2}^{N}$. Where $N$ is the number of pixels in the image. In our experiment the number of decomposition levels were set to 4 .

2. Estimate the noise from the detail coefficients. In this paper we used global threshold and level dependent threshold. Global threshold is calculated from the HH band coefficients of level 4. In level dependent threshold we calculated the threshold from the $\mathrm{HH}$ band of that particular level. In this paper we used the universal 
threshold which is a simple entropy measure totally depends on the size of the signal $T=\sigma \cdot \sqrt{2 \log (N)}$ where $N$ is the number of pixels in the image and $T$ is the threshold value. The noise level is estimated using the method developed by Jhonson and Donoho in the wavelet domain and suggested a robust estimate that is based only on the empirical wavelet coefficients at the finest resolution level. The reason for considering only the finest level is that the corresponding empirical wavelet coefficients tend to consist mostly of noise. Since there is some signal present even at this level, Donoho and Johnstone proposed a robust estimate of the noise level using the following equation [15],[16],[17],[14].

$$
\sigma(\operatorname{mad})=\frac{\operatorname{median}\left\{\left|w_{j}\right|: j=1,2, \ldots \frac{N}{2}\right\}}{0.6745}
$$

here $w 0, w 1, w 2 \ldots$ are the detail coefficients in the finest level. using $\sigma$ and $T$ we will continue to the next step wavelet shrinkage.

3. Apply the shrinkage operation on the wavelet coefficients. Shrinkage operation is performed on the wavelet coefficients usin the threshold estimated in the previous step. In this paper we used hard thresholding, soft thresholding, semi-soft thresholding and stein thresholding.hard thresholding method zeros the coefficients that are smaller than the threshold and leaves the other ones unchanged. In contrast, the soft thresholding scales the remaining coefficients in order to form a continuous distribution of the coefficients centered on zero.Several varieties of soft thresholding are described in the literature [18],[19],[20],[21],[14].

Hard Thresholding:

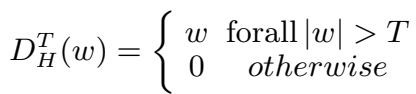

Soft Thresholding:

$$
D_{H}^{s}(w)=\operatorname{sgn}(w) \max (0 ;|w|-T)
$$

Semi-soft thresholding is a familly of non-linearities that interpolates between soft and hard thresholding.It uses both a main threshold $T$ and a secondary threshold $T 1=\mu * T$.

$$
D_{S S}^{T T 1}(w)=\left\{\begin{array}{cc}
0 & |w| \leq T \\
\operatorname{sgn}(w) \frac{T 1(|w|-T)}{T 1-T} & T<|w| \leq T 1 \\
w & |w|>T 1
\end{array}\right.
$$

When $\mu=1$ the semi-soft thresholding performs a hard thresholding, whereas when $\mu=\infty$, it performs a soft thresholding. Stein Thresholding: Another way to achieve a trade-off between hard and soft thresholding is to use a soft-squared thresholding non-linearity, also named a Stein estimator.

4. Apply the inverse transform to get the denoised image. In our paper we used inverse DWT, inverse UDWT and Inverse Dual Tree Complex Tree wavelet transforms on the approximation and detail coefficients to get the denoised image.

\section{RESULTS AND DISCUSSIONS}

\subsection{Experimental Setup}

Forward Transform: DWT and UDWT with 'db4' family, symlet family. DTCWT developed by Ivan W Selesnick.

Number of Decomposition Levels: 4
Shrinkage Functions: Hard, Soft, Semi-soft

Inverse Transform: IDWT and IUDWT with 'db4' family, symlet family. Inverse DTCWT developed by Ivan W Selesnick.

Quality Metrics: Mean Square Error (MSE),Peak Signal to Noise Ratio(PSNR), Structural Similarity Index Measure(SSIM) etc. [22],[23].

\subsection{Quality Metrics}

The following image quality metrics were used to evaluate the performance of the various wavelet transforms.

6.2.1 Mean Square Error(MSE). The mean square error between a image $f(x, y)$ and denoised image $\hat{f}(x, y)$ is given as

$$
M S E=\frac{1}{M N} \sum_{x=1}^{M} \sum_{y=1}^{N}[\hat{f}(x, y)-f(x, y)]^{2}
$$

6.2.2 Peak Signal to Noise Ratio(PSNR). PSNR is the peak signal-to-noise ratio in decibels $(\mathrm{dB})$. The PSNR is only meaningful for data encoded in terms of bits per sample, or bits per pixel. For example, an image with 8 bits per pixel contains integers from 0 to 255 .

$$
P S N R=20 \log _{10}\left(\frac{2^{B}-1}{\sqrt{M S E}}\right)
$$

Where B represents bits per sample.

6.2.3 The structural similarity (SSIM). This index is a method for measuring the similarity between two images. The SSIM index is a full reference metric, in other words, the measuring of image quality based on an initial uncompressed or distortion-free image as reference. SSIM is designed to improve on traditional methods like peak signal-to-noise ratio (PSNR) and mean squared error (MSE), which have proved to be inconsistent with human eye perception.

The SSIM metric is calculated on various windows of an image. The measure between two windows $x$ and $y$ of common size $N N$ is

$$
\operatorname{SSIM}(x, y)=\frac{\left(2 \mu_{x} \mu_{y}+c_{1}\right)\left(2 \sigma_{x y}+c_{2}\right)}{\left(\mu_{x}^{2}+\mu_{y}^{2}+c_{1}\right)\left(\sigma_{x}^{2}+\sigma_{y}^{2}+c_{2}\right)}
$$

$\mu_{x}$ is the average of $x$ and $\mu_{y}$ is the average of $y$.

$\sigma_{x}^{2}$ is the variance of $x$ and $\sigma_{y}^{2}$ is the variance of $y$.

$\sigma_{x y}$ is the covariance of $x$ and $y$.

$c_{1}=\left(k_{1} L\right)^{2}, c_{2}=\left(k_{2} L\right)^{2}$ two variables to stabilize the division with weak denominator.

$L$ is the dynamic range of the pixel-values.

$k_{1}=0.01$ and $k_{2}=0.03$ by default.

The resultant SSIM index is a decimal value between -1 and 1 , and value 1 is only reachable in the case of two identical sets of data. Typically it is calculated on window sizes of $8 \times 8$. The window can be displaced pixel-by-pixel on the image.

\subsubsection{Maximum Difference. Maximum difference is defined as}

$$
M D=\max (|f(x, y)-\hat{f}(x, y)|)
$$

The large value of maximum difference means de-noised image is poor quality. 
6.2.5 Normalised Absolute Error (NAE). The large value of normalised absolute error means that de-noised image is poor quality and is defined as

$$
N A E=\frac{\sum_{x=0}^{M-1} \sum_{y=0}^{N-1}|f(x, y)-\hat{f}(x, y)|}{\sum_{x=0}^{M-1} \sum_{y=0}^{N-1}|f(x, y)|}
$$

6.2.6 Signal To Noise Ratio (SNR). Signal to noise ratio in an image is calculated as

$$
S N R=\frac{\mu}{\sigma}
$$

Where $\mu$ is the average information in the signal and $\sigma$ is the standard deviation of the signal which represents the amount of noise present in the image.

6.2.7 Structural Content. For an $M x N$ image the structural content is defined as

$$
S C=\frac{1}{K} \sum_{k=1}^{K} \frac{\sum_{x=0}^{M-1} \sum_{y=0}^{N-1} f_{k}(x, y)^{2}}{\sum_{x=0}^{M-1} \sum_{y=0}^{N-1} \hat{f}_{k}(x, y)^{2}}
$$

\section{3 observations}

The tabulations were made for DWT, UDWT and Dual Tree Complex wavelets and three shrinkage functions as shown in the following tables.

The performance of the proposed filters is compared with the traditional spatial filters. If the PSNR value is high it does not mean that the image is denoised in better way. Even the noise is removed it suffers from blurring and ringing effects when DWT is used. These artifacts are eliminated by using Dual tree Complex Wavelet Transform in place of DWT. The denoised images were shown in the following figures.

Table 1. : Performance of Spatial Filters

\begin{tabular}{rrrr}
\hline Metric & Wiener & Homogeneous Mask Area & Geometric \\
\hline MSE & 145.1333 & 575.7459 & 499.2489 \\
SNR & 16.9359 & 10.6931 & 11.2321 \\
PSNR & 29.5234 & 23.5388 & 24.1579 \\
SSIM & 0.7915 & 0.5703 & 0.6714 \\
SC & 1.0944 & 1.2405 & 1.2762 \\
MD & 61 & 146 & 217 \\
NAE & 0.2071 & 0.3754 & 0.3399 \\
\hline
\end{tabular}

From the above results it is observed that spatial filtering is introducing blurring while denoising the images. The DWT is introducing checker board artifacts when the image is heavily corrupted with the speckle. The undecimated wavelet transform is performing better than the spatial filters and DWT but more computational complexity is involved while denoising the image. The structural
Table 2. : Performance of Discrete Wavelet Transform

\begin{tabular}{rrrr}
\hline Metric & Hard Threshold & Soft Threshold & Semi-Soft Threshold \\
\hline MSE & 330.9807 & 565.463 & 744.2902 \\
SNR & 13.168 & 10.9319 & 11.0001 \\
PSNR & 25.9431 & 23.6171 & 22.4237 \\
SSIM & 0.7812 & 0.5904 & 0.7566 \\
SC & 1.1952 & 1.1656 & 0.664 \\
MD & 203 & 119 & 240 \\
NAE & 0.2301 & 0.3831 & 0.3118
\end{tabular}

Table 3. : Performance of Undecimated Discrete Wavelet Transform

\begin{tabular}{rrrr}
\hline Metric & Hard Threshold & Soft Threshold & Semi-Soft Threshold \\
\hline MSE & 273.2872 & 582.8712 & 533.4604 \\
SNR & 13.7038 & 10.6961 & 11.0521 \\
PSNR & 25.3562 & 23.0666 & 23.4514 \\
SSIM & 0.6354 & 0.618 & 0.6351 \\
SC & 0.9841 & 0.8685 & 0.8793 \\
MD & 172 & 184 & 183 \\
NAE & 0.2445 & 0.3661 & 0.3416 \\
\hline
\end{tabular}

Table 4. : Performance of Dualtree Complex Wavelet Transform

\begin{tabular}{rrrr}
\hline Metric & Hard Threshold & Soft Threshold & Semi-Soft Threshold \\
\hline MSE & 541.2894 & 539.2893 & 469.658 \\
SNR & 11.0123 & 11.0186 & 11.5688 \\
PSNR & 23.3881 & 23.4042 & 24.0046 \\
SSIM & 0.7489 & 0.8477 & 0.7979 \\
SC & 0.8705 & 0.8741 & 0.8934 \\
MD & 170 & 178 & 173 \\
NAE & 0.3405 & 0.3408 & 0.3082
\end{tabular}

content is preserved in undecimated wavelet transform based denoising. Finally the dual tree complex wavelet transform is having low computational complexity and good capability of preserving edges in the denoised images. So the carotid artery image denoising where edge information is very important it is useful to use dual tree complex wavelet transform based filters than spatial filtering and DWT filtering.

\section{REFERENCES}

[1] A. Bovik, Handbook of Image and Video Processing. Communications, Networking and Multimedia, Elsevier Science, 2010.

[2] R. C. Gonzalez and R. E. Woods, Digital Image Processing (3rd Edition). Upper Saddle River, NJ, USA: Prentice-Hall, Inc., 2006.

[3] R. Rangayyan, Biomedical Image Analysis. Biomedical Engineering, CRC Press, 2004.

[4] V. S. Frost, J. A. Stiles, K. S. Shanmugan, and J. C. Holtzman, "A model for radar images and its application to adaptive digital filtering of multiplicative noise," Pattern Analysis and Machine Intelligence, IEEE Transactions on, vol. PAMI4, pp. 157-166, march 1982.

[5] R. M. Rangayyan, M. Ciuc, and F. Faghih, "Adaptiveneighborhood filtering of images corrupted by signaldependent noise," Appl. Opt., vol. 37, pp. 4477-4487, Jul 1998. 


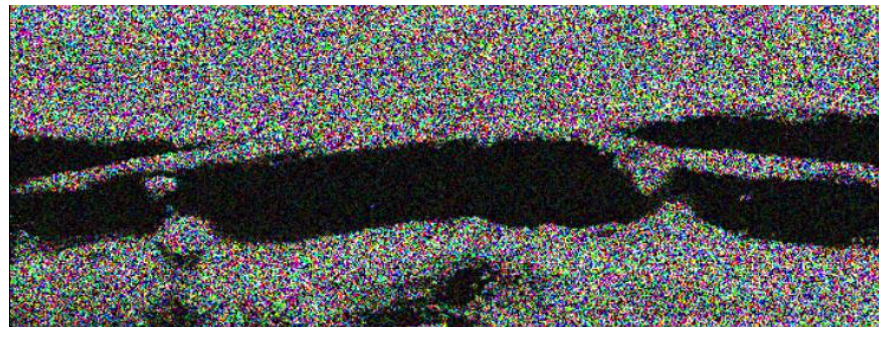

Fig. 4: Carotid artery image corrupted with speckle noise

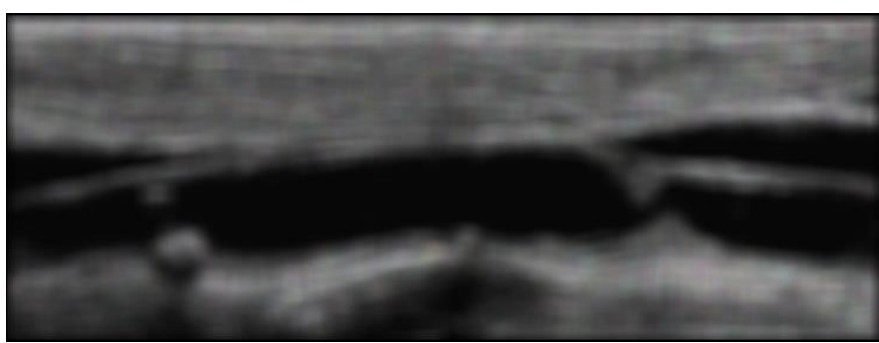

Fig. 5: Denoised Image with Geometric Spatial filter

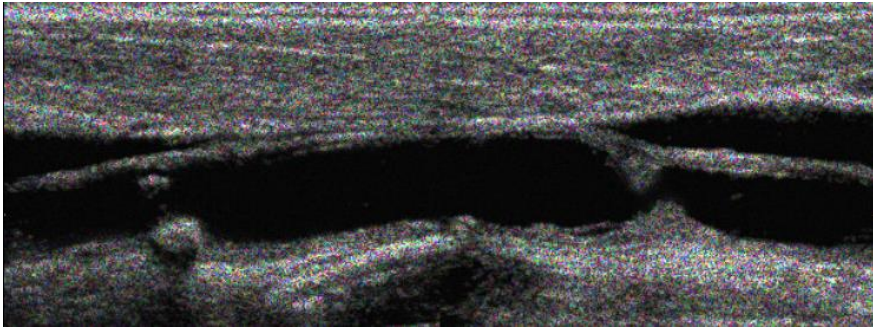

Fig. 6: Denoised Image with Discrete Wavelet Transforms with semisoft thresholding

[6] R. B. Paranjape, R. M. Rangayyan, and W. M. Morrow, "Adaptive neighborhood mean and median image filtering," Journal of Electronic Imaging, vol. 3, no. 4, pp. 360-367, 1994.

[7] M. I. Sezan and A. M. Tekalp, "Survey of recent developments in digital image restoration," Optical Engineering, vol. 29, no. 5, pp. 393-404, 1990.

[8] R. M. Rangayyan and A. Das, "Filtering multiplicative noise in images using adaptive region-based statistics," Journal of Electronic Imaging, vol. 7, no. 1, pp. 222-230, 1998.

[9] M. A. Schulze, "Edge-enhancing nonlinear filter for reducing multiplicative noise," pp. 46-56, 1997.

[10] A. I. Qureshi, O. Saeed, and A. A. Malik, "Abstract 85: Is progressively worsening severity of internal carotid artery stenosis by carotid doppler ultrasound in asymptomatic patients a risk factor for ischemic stroke?," vol. 49, no. Suppl 1, pp. A85-A85, 2018.

[11] G. Strang and T. Nguyen, Wavelets and Filter Banks. Wellesley-Cambridge Press, 1996.

[12] J. L. Starck, J. Fadili, and F. Murtagh, "The undecimated wavelet decomposition and its reconstruction," IEEE Transactions on Image Processing, vol. 16, pp. 297-309, Feb 2007.

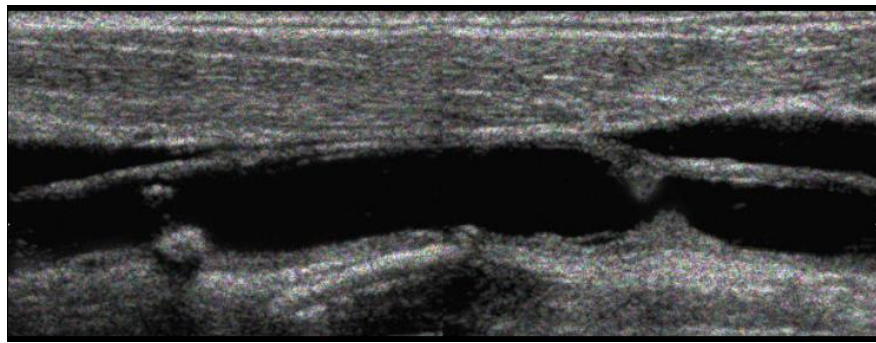

Fig. 7: Denoised Image with Complex Discrete Wavelet Transforms with semisoft thresholding

[13] I. W. Selesnick, R. G. Baraniuk, and N. C. Kingsbury, "The dual-tree complex wavelet transform," IEEE Signal Processing Magazine, vol. 22, pp. 123-151, Nov 2005.

[14] V. N. P. Raj and T. Venkateswarlu, "Denoising of medical images using dual tree complex wavelet transform," Procedia Technology, vol. 4, pp. 238 - 244, 2012. 2nd International Conference on Computer, Communication, Control and Information Technology( C3IT-2012) on February 25 - 26, 2012.

[15] F. Russo, "An image-enhancement system based on noise estimation," Instrumentation and Measurement, IEEE Transactions on, vol. 56, pp. 1435 -1442, aug. 2007.

[16] S. Aja-Fernndez, G. Vegas-Snchez-Ferrero, M. MartnFernndez, and C. Alberola-Lpez, "Automatic noise estimation in images using local statistics. additive and multiplicative cases," Image and Vision Computing, vol. 27, no. 6, pp. $756-$ 770, 2009.

[17] D. L. Donoho, I. M. Johnstone, G. Kerkyacharian, and D. Picard, "Wavelet shrinkage: Asymptopia?," Journal of the Royal Statistical Society. Series B (Methodological), vol. 57, no. 2, pp. 301-369, 1995.

[18] S.-M. Yang and S.-C. Tai, "A design framework for hybrid approaches of image noise estimation and its application to noise reduction," Journal of Visual Communication and Image Representation, vol. 23, no. 5, pp. 812 - 826, 2012.

[19] G. Andria, F. Attivissimo, G. Cavone, N. Giaquinto, and A. Lanzolla, "Linear filtering of 2-d wavelet coefficients for denoising ultrasound medical images," Measurement, vol. 45, no. 7, pp. $1792-1800,2012$.

[20] S. G. Chang, B. Yu, and M. Vetterli, "Adaptive wavelet thresholding for image denoising and compression," IEEE TRANSACTIONS ON IMAGE PROCESSING, vol. 9, no. 9, pp. 15321546, 2000.

[21] C. P. Loizou, C. S. Pattichis, C. I. Christodoulou, R. S. H. Istepanian, M. Pantziaris, and A. Nicolaides, "Comparative evaluation of despeckle filtering in ultrasound imaging of the carotid artery," IEEE Transactions on Ultrasonics, Ferroelectrics, and Frequency Control, vol. 52, pp. 1653-1669, Oct 2005.

[22] Z. Wang, A. C. Bovik, H. R. Sheikh, and E. P. Simoncelli, "Image quality assessment: from error visibility to structural similarity," IEEE Transactions on Image Processing, vol. 13, pp. 600-612, April 2004.

[23] C. Loizou, C. Pattichis, R. Istepanian, M. Pantziaris, E. Kyriakou, T. Tyllis, and A. Nicolaides, "Ultrasound image quality evaluation," in Information Technology Applications in Biomedicine, 2003. 4th International IEEE EMBS Special Topic Conference on, pp. 138 - 141, april 2003. 